\title{
A retrospective study of changes in skin cancer characteristics over 11 years
}

\author{
Young Ji Park, \\ Gyu Hyeon Kwon, \\ Jun Oh Kim, \\ Nam Kyun Kim, \\ Woo Sang Ryu, \\ Kyung Suk Lee \\ Department of Plastic and \\ Reconstructive Surgery, Gyeongsang \\ National University Hospital, Jinju, \\ Korea
}

\begin{abstract}
Background: The incidence of skin cancer, which is primarily caused by exposure to ultraviolet radiation, has steadily increased in recent years. The authors of the present study sought to investigate changes in the epidemiology of skin cancer by conducting a retrospective review of patients diagnosed with skin cancer who received related care at a single medical institution.

Methods: The present study included patients who were diagnosed with skin cancer and received treatment at Gyeongsang National University Hospital from 2008 to 2018. The site and type of skin cancer, the number of patients with skin cancer each year, the sex and sex ratio of the patients, and changes in patients' age at first diagnosis were examined through retrospective chart reviews.

Results: The number of patients with skin cancer significantly increased, but statistically significant changes were not found in patients' sex, skin cancer sites, or the types of skin cancer. However, patients' age at the first diagnosis of skin cancer showed a statistically significant decrease starting in 2015.

Conclusion: In this study, the number of patients with skin cancer increased over time. However, patients' age at first diagnosis has decreased since 2015. Therefore, younger patients should take care to prevent skin cancer, and further research on the causes of skin cancer in younger patients is needed.
\end{abstract}

Keywords: Carcinoma, basal cell / Carcinoma, squamous cell / Epidemiology / Prevalence / Skin neoplasms

\section{INTRODUCTION}

The incidence of skin cancer has steadily increased, concomitantly with changes in society and medical care [1]. Ultraviolet radiation (UVR) is regarded as the most significant risk factor for skin cancer [2]. Since in humans, the face and neck are the body parts most often exposed to UVR, they have generally been reported as the most frequent sites of skin cancer occurrence [3].

\section{Correspondence: Kyoung Suk Lee}

Department of Plastic and Reconstructive Surgery, Gyeongsang National

University Hospital, 79 Gangnam-ro, Jinju 52727, Korea

E-mail: opensound@hanmail.net

Received January 14, 2020 / Revised February 28, 2020 / Accepted April 20, 2020
Basal cell carcinoma (BCC) and squamous cell carcinoma (SCC) have been designated as the major subtypes of skin cancer. According to a study conducted by the US National Institutes of Health, $70 \%-80 \%$ of skin cancer patients have BCC, whereas approximately $20 \%$ have SCC. Other skin cancers, such as Merkel cell carcinoma and diverse sarcomas, account for less than $1 \%$ of the entire incidence of skin cancer $[4,5]$. An increasing incidence of skin cancer has been reported in Western countries, while other studies have reported a stable incidence of skin cancer in Asian countries. In the United States, 2,152,500 patients were estimated to have been diagnosed with skin cancer in 2006 [5-8]. Skin cancer affects men and aged people more frequently, although in recent years, it has become 
more common for younger individuals to develop skin cancer because of their increasing exposure to UVR $[9,10]$. The average age at the first diagnosis of skin cancer was reported to be approximately 64 years, and this age has gradually increased over time $[11,12]$. Several studies, including that of Chung et al. [13] on the epidemiology of skin cancer, have explored this issue in Korea [14-20]. However, previous studies have mostly focused on describing trends in the incidence of skin cancer without statistical analyses. Therefore, the present study analyzed patients who were diagnosed with skin cancer and received treatment at the Department of Plastic Surgery of Gyeongsang National University Hospital during an 11-year period to provide information to support the better diagnosis and treatment of patients with skin cancer.

\section{METHODS}

In total, 662 patients who were diagnosed with skin cancer and received treatment at the Department of Plastic Surgery of Gyeongsang National University Hospital were included in the present study, which aimed to identify changes in the epidemiology of skin cancer from 2008 to 2018. Retrospective chart reviews were conducted to collect information on patients' sex and sex ratio, age at the first skin cancer diagnosis, and sites and types of skin cancer.

Statistical analyses of all data were carried out using SAS version 9.4 (SAS Institute, Cary, NC, USA). The sites of skin cancer occurrence were broadly classified into the face, extremities, and trunk, and multinomial logistic regression analysis was used to evaluate changes in the distribution of sites over time. To examine skin cancer that occurred on the face in more detail, sites on the face were subdivided into the scalp, forehead, cheek, chin, neck, periorbital region, ear, nose, upper lip, lower lip, and neck, and the proportions of incident skin cancers at each site were compared with the results reported by Kim et al. [19]. Only skin cancer was included in the present study, and premalignant skin lesions were excluded. Skin cancer was categorized into four types: BCC, SCC, malignant melanoma (MM), and others (adenocarcinoma, angiosarcoma, extramammary Paget disease, Merkel cell carcinoma, and verrucous carcinoma). Multinomial logistic regression analysis was conducted to analyze differences in the occurrence of the different types of skin cancer. Changes in the annual number of patients diagnosed with skin cancer were investigated and the significance of changes in the number of patients was tested using Poisson regression analysis. The total number of male patients was divided by the number of female patients in each year to obtain the sex ratio (male/female, \%) and the significance of changes in the sex ratio over time was analyzed using the chisquare test. Patients' age at the first diagnosis of skin cancer was examined, and the significance of changes in this parameter was analyzed using the Kruskal-Wallis test.

\section{RESULTS}

The present retrospective study included 662 patients who resided in South Gyeongsang Province, were diagnosed with skin cancer at the Department of Plastic Surgery of Gyeongsang National University Hospital, and received treatment during the period from January 1, 2008 to December 31, 2018. The overwhelming majority of cases of skin cancer occurred on the face $(n=567 ; 85.65 \%)$, followed by the extremities $(n=60 ; 9.06 \%)$ and the trunk ( $\mathrm{n}=35 ; 5.29 \%)$. A $p$-value of 0.6863 was found, indicating that there was no statistically significant change in the sites of skin cancer incidence. Skin cancers on the face were categorized according to whether they were found on the scalp, forehead, cheek, chin, neck, periorbital region, ear, nose, upper lip, or lower lip, as in a previous study. In this study, the cheek accounted for the largest proportion of cases $(n=190 ; 33.5 \%)$, followed by the forehead ( $\mathrm{n}=145 ; 25.6 \%)$, and the nose $(\mathrm{n}=80$; 14.1\%) (Fig. 1). BCC was the most common type of skin cancer $(\mathrm{n}=344 ; 51.96 \%)$, followed by SCC $(\mathrm{n}=267 ; 40.33 \%)$, other skin cancers $(n=30 ; 4.53 \%)$, and MM $(n=21 ; 3.17 \%)$. Multinomial logistic regression was conducted to determine whether any significant changes in the types of skin cancer occurred over time. A $p$-value of 0.0572 was obtained, implying that no significant change took place. The highest number of patients $(\mathrm{n}=106 ; 16.01 \%)$ was diagnosed in 2018, followed by 2015 $(\mathrm{n}=88 ; 13.29 \%)$ and $2016(\mathrm{n}=75 ; 11.33 \%) . \mathrm{A} p$-value of

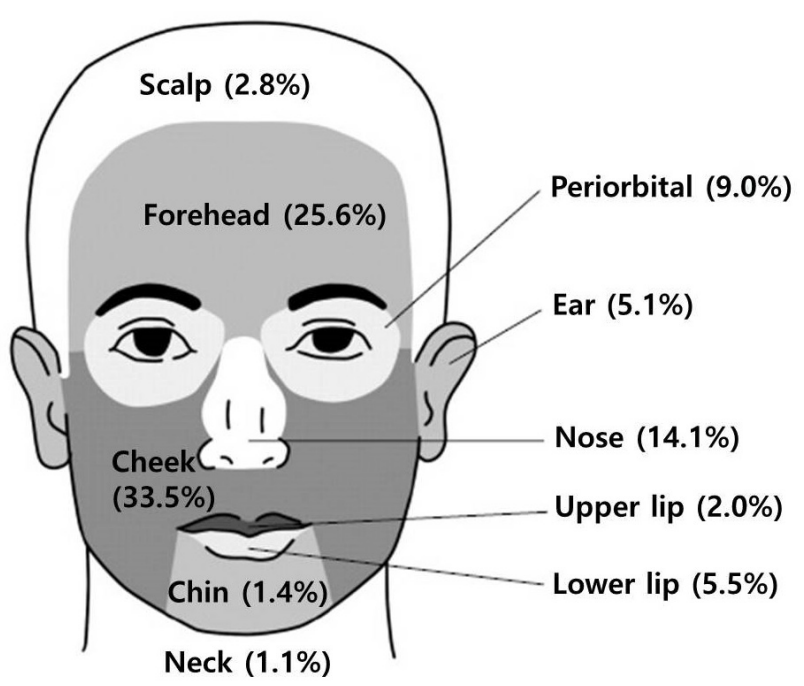

Fig. 1. Distribution of skin cancer sites on the face. 
No. of patients diagnosed with skin cancer from 2008 to 2018

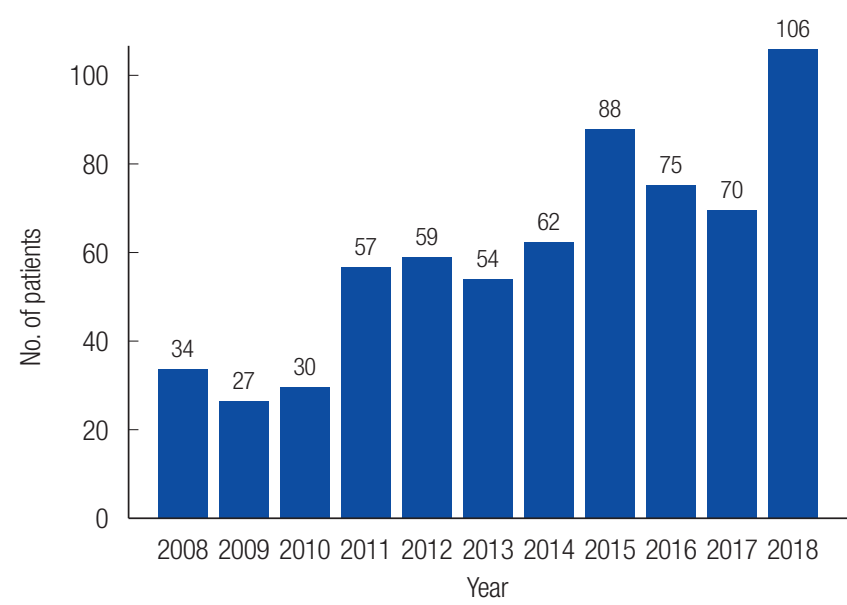

Fig. 2. Changes in the number of patients diagnosed with skin cancer over time (results of Poisson regression analysis, $p<0.0001$ ).

$<0.0001$ was obtained, indicating a significant increase in the number of patients over time (Fig. 2). The sex ratio (male/female, \%) of patients with skin cancer was also analyzed. There were 256 male patients and 406 female patients, resulting in a sex ratio of $63.05 \%$, corresponding to a more frequent incidence of skin cancer in women than in men. A $p$-value of 0.4528 was obtained, indicating no statistically significant change in the sex ratio. The average age at the first diagnosis of skin cancer was 76.2 years in 2014, which was the highest age during the study period. The average age remained similar from 2008 to 2014 and then gradually decreased from 2015 to 2018 . A pvalue of 0.0484 was obtained, indicating a significant downward trend in age at the first diagnosis of skin cancer (Fig. 3).

\section{DISCUSSION}

A retrospective chart review was conducted of patients living in South Gyeongsang Province who were diagnosed with skin cancer and received treatment at Gyeongsang National University Hospital during the 11-year period from 2008 to 2018 . The aim of this study was to examine trends in the incidence of skin cancer and characteristics of skin cancer patients.

A statistical analysis of trends in the sites of skin cancer incidence indicated that no statistically significant changes had taken place. This result is similar to those of previous studies, including the study conducted by Jung and Kim [2], in which UVB radiation was identified as the major cause of skin cancer and the most frequently observed site of skin cancer was the face, the body part most frequently exposed to UVB radiation. In their study, no additional changes in the trends of the incidence of skin cancer were found. In addition, the incidence of
Age of patients diagnosed with skin cancer from 2008 to 2018

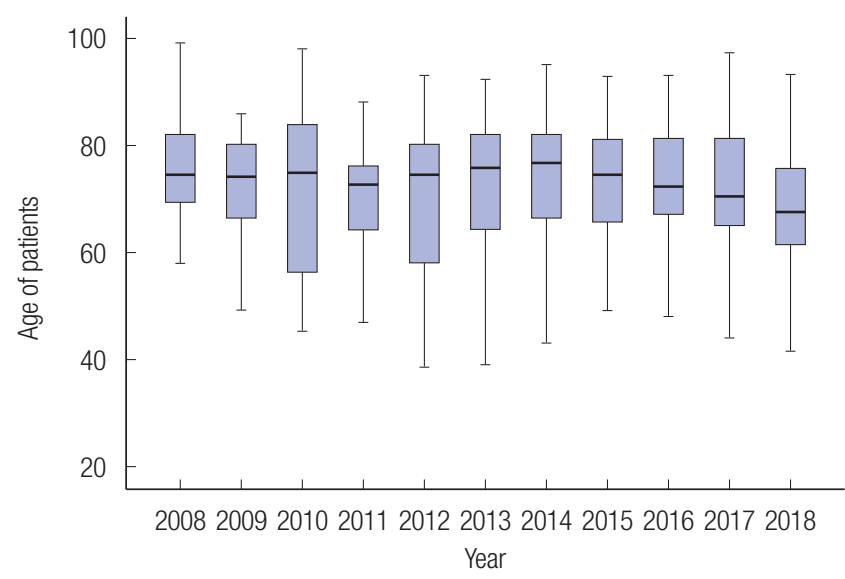

Fig. 3. Changes in patients' age at first diagnosis over time (results of the Kruskal-Wallis test, $p=0.0484$ ).

skin cancer on the face was similar to the results of Kim et al. [12], who reported that the cheek was the most frequent site of skin cancer, followed by the forehead and the nose. An explanation for this result is that the cheeks and forehead form large areas of the face, whereas the relatively high frequency of incidence of skin cancer on the nose is ascribable to more intense contact with UVR due to the protrusion of the nose from the center of the face. The types of skin cancer occurring on the face were classified into BCC, SCC, MM, and others (adenocarcinoma, angiosarcoma, extramammary Paget disease, Merkel cell carcinoma, and verrucous carcinoma). Kim et al. [12] reported that the most common type of skin cancer was BCC (46.2\%), followed by SCC (27.6\%), and MM (19.1\%), a distribution similar to that found in the present study, where BCC accounted for the majority of cases (52.0\%), followed by SCC (40.33\%), and MM (3.17\%). Thus, the order of the incidence of different types of skin cancer was identical between these two studies, although the frequency of incidence of SCC was somewhat higher in the present study and that of MM was somewhat lower. We presume that the relatively low incidence of MM in the present study could be attributable to the smaller population of patients diagnosed with MM.

Previous studies have reported a significant increase in the number of patients diagnosed with skin cancer annually. Chun et al. [20] explored the incidence of skin cancer in patients residing in Gwangju Metropolitan City and South Jeolla Province in the 1980s and reported an increase in the incidence of skin cancer and premalignancies similar to the results of the present study. In another study [19], skin cancer patients in their 50s comprised over $95 \%$ of the total number of patients. According to the National Statistical Office [21], the population residing in 
South Gyeongsang Province stabilized starting in 2014, and began to decline in 2018. Despite the decrease in population, the significant increase in the annual number of skin cancer patients suggests an increase in the rate of early diagnosis, perhaps due to increasing attention paid by local residents to their health. Furthermore, population aging [22] may contribute to the increase in the number of patients with skin cancer, despite annual decreases in the overall population. Significant changes were not found in the sex or sex ratio of patients with skin cancer. In a study by $\mathrm{Oh}$ et al. [23] of people with skin cancer in Korea from 1999 to 2014, the sex ratio of patients with skin cancer was $57 \%$ males to $43 \%$ females. The results of the present study, which analyzed patients residing in South Gyeongsang Province, found a sex ratio of $63.05 \%$ males (256 male patients and 406 female patients). According to the National Statistical Office, the sex ratio (male/female, \%) of the population over 50 in South Gyeongsang Province was lower than that of the nation overall. Nonetheless, the fact that women accounted for a larger proportion of skin cancer cases than men in this study implies that female residents of South Gyeongsang Province may be at a higher incidence of skin cancer than women elsewhere in Korea, given the discrepancy with the results reported by Oh et al. [23].

Statistically significant changes in the age at first diagnosis were observed in the present study. The average age at diagnosis remained stable until 2014, and then gradually trended downward starting in 2015. In contrast, in an epidemiological study of skin cancer since 1980 [13-15], the average age at the first skin cancer diagnosis was reported to gradually increase. In the study conducted by Kim et al. [12] of 1,699 patients at eight hospitals, the average age of patients with skin cancer also gradually increased over time. The cause of this gradual increase was attributed to the increased number of aged patients owing to the increase in the average lifespan [22]. The gradual decrease in the average age at first diagnosis identified in the present study contrasts with the results of previous studies. However, more early diagnoses of skin cancer may have been made due to an increased number of younger people seeking care at hospitals, reflecting social trends for greater attention to be paid to health. However, this explanation may have limitations in explaining the decrease in the average age at the first diagnosis of skin cancer. Nonetheless, our findings indicate that surgeons caring for and treating young patients with skin lesions should be aware of the potential for skin cancer.

The present study had certain limitations. First, it was conducted at a single medical institution, and the retrospective review of patients with skin cancer encompassed a relatively short period of time (11 years). The types of skin cancer might have been biased by the relatively small size of the patient pool. Furthermore, a simple comparison was made with the epidemiological changes of skin cancer over time reported in a previous study.

However, the authors of the present paper focused on trends and changes of incidence of skin cancer rather than just epidemiologic analyses of skin cancer found in previous studies. This may contribute to the significance of the present study. The decrease in the average age at the first skin cancer diagnosis during the last 5 years of the study period was statistically significant. Thus, further studies are needed to investigate the decreasing trend in the annual average age at first diagnosis and its associated causes.

\section{NOTES}

\section{Conflict of interest}

No potential conflict of interest relevant to this article was reported.

\section{Ethical approval}

The study was approved by the Institutional Review Board of Gyeongsang National University Hospital (IRB No. 2019-09022) and performed in accordance with the principles of the Declaration of Helsinki.

\section{ORCID}

Young Ji Park https://orcid.org/0000-0001-8416-7833

Gyu Hyeon Kwon https://orcid.org/0000-0002-8377-4698

Jun Oh Kim https://orcid.org/0000-0002-3860-6433

Nam Kyun Kim https://orcid.org/0000-0002-1511-9106

Woo Sang Ryu https://orcid.org/0000-0002-7472-3813

Kyung Suk Lee https://orcid.org/0000-0001-7731-1761

\section{REFERENCES}

1. Glanz K, Schoenfeld ER, Steffen A. A randomized trial of tailored skin cancer prevention messages for adults: Project SCAPE. Am J Public Health 2010;100:735-41.

2. Jung $\mathrm{YH}$, Kim SS. A clinical study in malignant skin tumors. J Korean Soc Plast Reconstr Surg 1982;9:377-88.

3. Hwang JI, Kim HS, Park H, Kim JW, Yu DS. A statistical survey of major cutaneous malignant tumors for the last 10 years (2000-2010, North-east Gyeonggido Province). Korean J Dermatol 2011;49:97-105.

4. Athas WF, Hunt WC, Key CR. Changes in nonmelanoma skin cancer incidence between 1977-1978 and 1998-1999 in Northcentral New Mexico. Cancer Epidemiol Biomarkers Prev 
2003;12:1105-8.

5. Demers AA, Nugent Z, Mihalcioiu C, Wiseman MC, Kliewer EV. Trends of nonmelanoma skin cancer from 1960 through 2000 in a Canadian population. J Am Acad Dermatol 2005;53: 320-8.

6. Lomas A, Leonardi-Bee J, Bath-Hextall F. A systematic review of worldwide incidence of nonmelanoma skin cancer. $\mathrm{Br} J$ Dermatol 2012;166:1069-80.

7. Erdmann F, Lortet-Tieulent J, Schuz J, Zeeb H, Greinert R, Breitbart EW, et al. International trends in the incidence of malignant melanoma 1953-2008-are recent generations at higher or lower risk? Int J Cancer 2013;132:385-400.

8. Birch-Johansen F, Jensen A, Mortensen L, Olesen AB, Kjaer SK. Trends in the incidence of nonmelanoma skin cancer in Denmark 1978-2007: rapid incidence increase among young Danish women. Int J Cancer 2010;127:2190-8.

9. Bath-Hextall F, Leonardi-Bee J, Smith C, Meal A, Hubbard R. Trends in incidence of skin basal cell carcinoma: additional evidence from a UK primary care database study. Int J Cancer 2007;121:2105-8.

10. Boi S, Cristofolini M, Micciolo R, Polla E, Dalla Palma P. Epidemiology of skin tumors: data from the cutaneous cancer registry in Trentino, Italy. J Cutan Med Surg 2003;7:300-5.

11. Dacosta Byfield S, Chen D, Yim YM, Reyes C. Age distribution of patients with advanced non-melanoma skin cancer in the United States. Arch Dermatol Res 2013;305:845-50.

12. Kim HS, Cho EA, Bae JM, Yu DS, Oh ST, Kang H, et al. Recent trend in the incidence of premalignant and malignant skin lesions in Korea between 1991 and 2006. J Korean Med Sci 2010; 25:924-9.

13. Chung HG, Moon TK, Bang DS, Lee MG. Clinical observation of cutaneous malignant tumors and premalignant lesions over 15 years (1982-1996). Korean J Dermatol 1999;37:1413-22.

14. Shin JH, Cho S, Whang KK, Hahm JH. An epidemiologic analysis of cutaneous malignant tumors over 15 years (1984-
1998). Korean J Dermatol 1999;37:1743-51.

15. Seo JJ, Won YH, Kim SJ, Lee SC, Chun IK. A clinical observation of cutaneous malignant tumors over 10 years (1987-1996, Chonnam province). Korean J Dermatol 1998;36:812-9.

16. Moon SE, Cho KH, Hwang JH, Kim JA, Youn JI. A statistical study of cutaneous malignant tumors. Korean J Dermatol 1998;36:7-15.

17. Jeong KB, Kim HC, Shin DH, Choi JS, Kim KH. A clinical observation of cutaneous premalignant and malignant tumors. Korean J Dermatol 2002;40:924-31.

18. Seo PG, Moon SE, Cho KH. A statistical study of cutaneous malignant tumors (1996-2000). Korean J Dermatol 2002;40: 129-37.

19. Kim DH, Kwon IH, Cho KH. A statistical study of cutaneous malignant tumors (2001-2005). Korean J Dermatol 2008;46: 1581-7.

20. Chun JS, Yun SJ, Lee JB, Kim SJ, Won YH, Lee SC. A statistical observation on 1,430 cases of cutaneous malignant tumors over 20 years (1987-2006) in Gwangju city and Chonnam province. Korean J Dermatol 2009;47:667-73.

21. Korean Statistical Information Service (KOSIS). Regional population growth rate statistics every year since 2014 [Internet]. Daejeon: KOSIS [cited 2020 Apr 22]. Available from: http:// kosis.kr/statHtml/statHtml.do?orgId=101\&tblId=DT_1YL206 21\&conn_path=I2.

22. Korean Statistical Information Service (KOSIS). Elderly population statistics by region since August 2019 [Internet]. Daejeon: KOSIS [cited 2020 Apr 22]. Available from: http://kosis. $\mathrm{kr} /$ statHtml/statHtml.do?orgId=101\&tblId=DT_1YL20631\&c onn_path=I2.

23. Oh CM, Cho H, Won YJ, Kong HJ, Roh YH, Jeong KH, et al. Nationwide trends in the incidence of melanoma and nonmelanoma skin cancers from 1999 to 2014 in South Korea. Cancer Res Treat 2018;50:729-37. 